\title{
НЕЙРОСЕТЕВОЕ МОДЕЛИРОВАНИЕ ПРОЦЕССА ОХЛАЖДЕНИЯ ПОЛОСЫ НА СТАНЕ ГОРЯЧЕЙ ПРОКАТКИ
}

\author{
И. А. Седых, В. А. Истомин \\ Липецкий государственный технический университет
}

Поступила в редакцию 10.12.2018 г.

\begin{abstract}
Аннотация. В работе рассмотрен и смоделирован на основе нейронных сетей процесс охлаждения полосы на стане горячей прокатки металлургического производства. Данный процесс схематично изображен на рисунке с выделением главных функциональных участков. Пояснены основные термины, относящиеся к приведенному процессу металлургического производства. Показаны структурные схемы радиально-базисной нейронной сети и нейронной сети Такаги - Сугено - Канга, описаны гибридные алгоритмы их обучения. В блоке программирования Mathcad разработаны программы, реализующие математические модели рассмотренных нейронных сетей. Входные и выходные данные для программ соответственно считываются и сохраняются в файлах MicrosoftExcel.Проведен отбор входных и выходных переменных моделируемого процесса, выполнена линейная нормализация исходных данных. Сформированы обучающая и проверочная выборки с помощью запросов в программе Microsoft Access. Для данных моделей выполнен ряд вычислительных экспериментов, в ходе которых были получены оптимальные структура и параметры радиально-базисной нейронной сети и нейронной сети Такаги - Сугено - Канга. Проведено сравнение обученных нейронных сетей, приведены графики отклонений обучающей и проверочной выборок, а также результаты их обучения. Найдены среднеквадратическая и относительная ошибки сетей по денормализованным выходным данным. На основании выполненного анализа выделены основные особенности и отличия вышеуказанных нейронных сетей. Сделаны выводы по проделанной работе.

Ключевые слова: моделирование, радиально-базисные нейронные сети, нейронные сети Такаги - Сугено - Канга, обучение нейронных сетей, процесс охлаждения полосы, архитектура нейронной сети.
\end{abstract}

\section{ВВЕДЕНИЕ}

Прокатка из всех способов обработки металлов пользуется наибольшим распространением вследствие непрерывности процесса, высокой производительности и возможности получения изделий самой разнообразной формы и улучшенного качества. Режим охлаждения существенно влияет на качество готового проката и его расход. В зависимости от предъявляемых требований и химического состава применяют быстрое или медленное охлаждение после прокатки. Например, инструментальные стали У9-У12 охлаждают в воде. При медленном охлаждении в структуре сталей У9-У12 образуется карбидная

(с) Седых И. А., Истомин В. А., 2019 сетка, что не допускается техническими условиями. Между чистовой клетью и роликовыми барабанными моталками широкополосных станов полоса интенсивно охлаждается водой. Быстрое охлаждение полосы с 850-950 до 600-650 ${ }^{\circ} \mathrm{C}$ обеспечивает равномерную структуру и исключает выпадение свободного цементита. Большинство легированных сталей не допускает быстрого охлаждения. В процессе быстрого охлаждения в стали образуются поверхностные и внутренние трещины (флокены). С увеличением содержания углерода с 0,10 до $0,70 \%$ величина потерь металла в виде окалины составляет соответственно 3,30-2,30 \% при охлаждении на воздухе. При охлаждении металла после прокатки водой или водяной пылью потери в виде окалины не превышают 1,5 \% [1]. Для дости- 
жения такого результата требуется правильная настройка системы управления данным участком производства, которая позволяет получать на выходе значения температуры смотки металла, находящейся в пределах допустимого диапазона значений. Разработка имитационной модели рассматриваемого процесса даёт возможность прогнозировать температуру смотки полосы на реальном металлургическом производстве при изменении управляющих параметров.

В результате проведения анализа литературных источников по теме исследования установлено, что в [2] представлены тепловая и математическая модели горячекатаной полосы на отводящем рольганге. На основе полученных моделей автором разработан и внедрен в действующую систему управления установкой ламинарного охлаждения алгоритм управления охлаждением полосы на отводящем рольганге широкополосного стана горячей прокатки, в основу которого положен принцип адаптивного управления. Алгоритм поддерживает заданную температуру смотки, как по возмущению начальных условий, так и по отклонению управляемой величины.

Нейросетевая модель на стане горячей прокатки рассматривалась только с теоретической точки зрения в [3], где предложены методы и алгоритмы, с помощью которых возможна реализация нейросетевого моделирования системы ускоренного охлаждения проката. Приведены некоторые направления исследований, которые могут быть использованы при разработке пакета нейросетевой идентификации систем.

Целью данного исследования было создание нейросетевых моделей процесса охлаждения полосы на стане горячей прокатки металлургического производства на основе радиально-базисной (РБФ) и Такаги - Сугено -
Канга (ТСК) нейронных сетей в приложении Mathcad, с достаточной точностью прогнозирующих температуру смотки металла. Кроме того, целью работы является исследование сравнения моделирующих способностей четкой РБФ и нечеткой ТСК сетей, обучающихся по сходным гибридным алгоритмам.

\section{ОПИСАНИЕ ПРОЦЕССА ОХЛАЖДЕНИЯ ПОЛОСЫ}

Схематично на рис. 1 показан участок душирования металла на стане горячей прокатки. На данной схеме слева показана последняя клеть горячекатаного стана (а), после которого по отводящему рольгангу (6) (рольганг устройство в виде ряда роликов, размещенных на станине для транспортирования металла к прокатному стану, подачи его в валки, приема из валков и передвижения между клетями стана, а также к вспомогательному или отделочному оборудованию) сталь проходит через 80 секций, оборудованных соплами для подачи воды (в). Именно этот участок называется душирование, т. е. ускоренное охлаждение горячекатаного металла струями воды. Непосредственно перед и после этого участка располагаются соответственно пирометр температуры стали (г), выходящей из последней клети, и пирометр температуры смотки металла (д). Замер последнего производится перед смоткой. Участок начала смотки показан на схеме справа (е), куда сталь поступает по отводящему рольгангу после достижения оптимальной температуры для её смотки.

На основании этого процесса и знаний, полученных при наблюдениях за ним, peaлизуются математические моделиРБФ и ТСК нейронных сетей, для которых происходит подбор архитектуры и параметров [4-9]. Для обучения и тестирования каждой сети в бло-

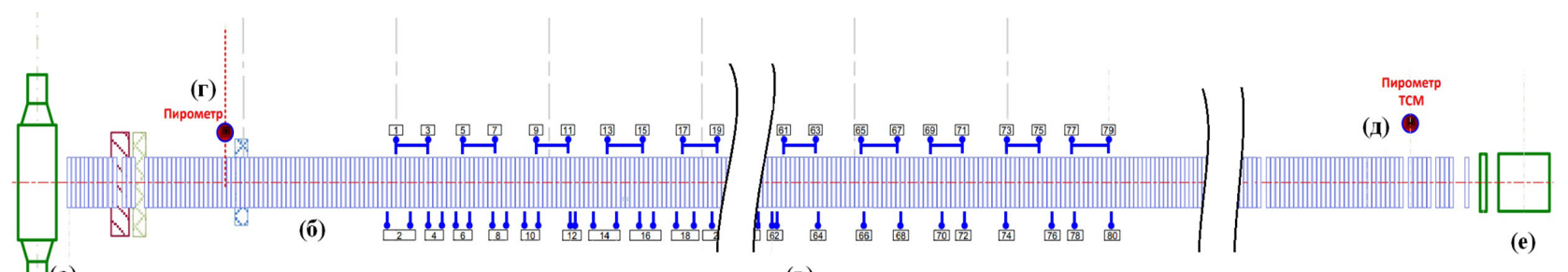

(в)

Рис. 1. Схема душирования 


\section{И. А. Седьцх, В. А. Истомин}

ке программирования пакета Mathcad были реализованы программы со следующими функциями: 1) для обучения нейронной сети по гибридному алгоритму[5]; 2) для продолжения обучения с последней сохранённой итерации в случаях непредвиденной остановки программы или долгого обучения; 3) для проверки обученной сети на контрольной выборке. Также в программах были реализованы подстройка шага обучения и постоянное сохранение сетей с наименьшим показателем ошибки за все итерации обучения. Входные и выходные данные для реализованных программ соответственно считываются и сохраняются в файлах Microsoft Excel. C помощью реализованных программ разработаны нейросетевые модели процесса охлаждения полосы на стане горячей прокатки.

\section{ГИБРИДНЫЕ АЛГОРИТМЫ ОБУЧЕНИЯ РАДИАЛЬНО-БАЗИСНЫХ И ТАКАГИ - СУГЕНО - КАНГА НЕЙРОННЫХ СЕТЕЙ}

Радиально-базисная сеть имеет фиксированную структуру с одним скрытым слоем и линейными выходными нейронами. Роль скрытых нейронов в ней играют базисные радиальные функции - специальный класс функций, значение которых монотонно уменьшается с увеличением расстояния входного вектора $X$ от центра $C$. Обобщенная структура нейронной сети РБФ представлена на рис. 2.

В качестве радиальных базисных функций $\varphi_{i}$ обычно используются функции Гаусса:

$$
\begin{aligned}
& \varphi_{i}\left(X_{L}\right)=\varphi_{i}\left(\left\|X_{L}-C_{i}\right\|\right)= \\
& =\exp \left(-\sum_{j=1}^{s X} \frac{\left\|x_{L, j}-C_{i, j}\right\|^{2}}{2 \sigma_{i, j}^{2}}\right),
\end{aligned}
$$

где $s X$ - количество входных переменных, $L=1, \ldots, o X ; o X-$ количество строк данных обучающей выборки, $i=1, \ldots, n ; n$ - количество нейронов на скрытом слое, $\sigma_{i, j}$ - ширина разброса, $C_{i, j}$ - центр функции активации $\varphi_{i}$ для $j$ входной переменной.

Роль нейронов выходного слоя сводится к взвешенному суммированию сигналов, генерируемых нейронами скрытого слоя:

$$
y_{L}=\sum_{i=1}^{n} w_{i} \cdot \varphi_{i}\left(X_{L}\right)
$$

где $w_{i}$ - это линейный параметр сети.

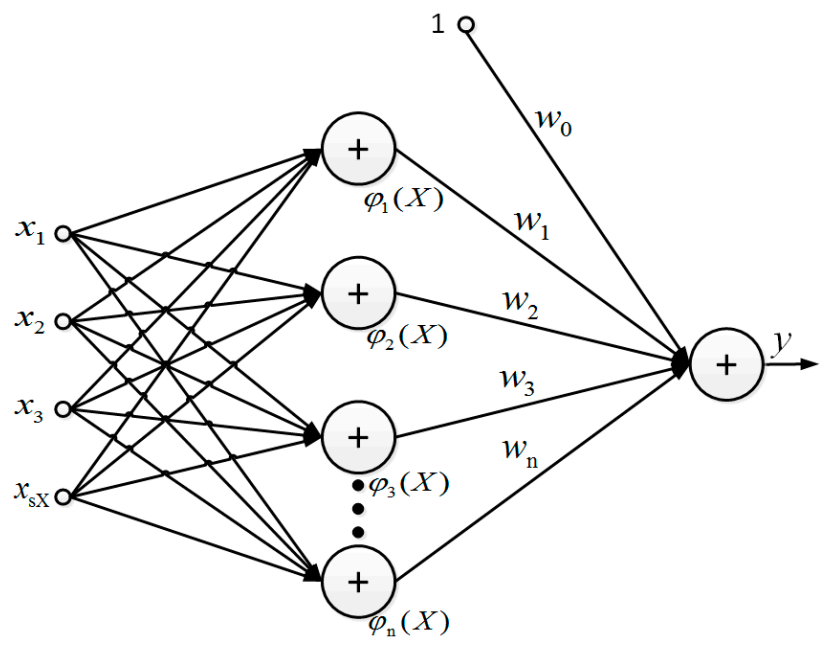

Рис. 2. Обобщённая структура нейронной сети РБФ

В работе рассматривается двухэтапный гибридный алгоритм обучения РБФ-сетей.

Рассмотрим первый этап гибридного алгоритма обучения РБФ. Сначала случайным образом задаются центры $C_{i, j}$ и $\sigma_{i, j}$, где $i=1, \ldots, n ; j=1, \ldots, s X$.

Далее составляются следующие матрицы:

$$
\begin{gathered}
G=\left[\begin{array}{ccccc}
1 & \varphi_{1}\left(X_{1}\right) & \varphi_{2}\left(X_{1}\right) & \cdots & \varphi_{n}\left(X_{1}\right) \\
1 & \varphi_{1}\left(X_{2}\right) & \varphi_{2}\left(X_{2}\right) & \cdots & \varphi_{n}\left(X_{2}\right) \\
\cdots & \cdots & \cdots & \cdots & \cdots \\
1 & \varphi_{1}\left(X_{o X}\right) & \varphi_{2}\left(X_{o X}\right) & \cdots & \varphi_{n}\left(X_{o X}\right)
\end{array}\right], \\
W=\left[\begin{array}{c}
w_{0} \\
w_{1} \\
\cdots \\
w_{n}
\end{array}\right], \quad Y=\left[\begin{array}{c}
y_{1} \\
y_{2} \\
\cdots \\
y_{o X}
\end{array}\right],
\end{gathered}
$$

где $X_{L}-L$ строка входных данных, $L=1 \ldots o X$.

Затем составляется система: $G \cdot W=Y$ и находится её псевдорешение:

$$
W=G^{+} \cdot Y \text {, }
$$

где $G^{+}$- это матрица, псевдообратная к матрице $G$.

Далее находится ошибка обучения РБФ:

$$
E=\frac{1}{2} \sum_{L=1}^{o X}\left(\hat{y}_{L}-y_{L}\right)^{2},
$$


где $\hat{y}_{L}$ - выходные значения нейронной сети, $y_{L}$ - фактическое выходное значение для $L$-й строки данных.

На втором этапе гибридного алгоритма обучения РБФ происходит пересчет центров и ширины разброса активационных функций. В результате дифференцирования функции (1) получаются следующие формулы [5]:

$$
\begin{aligned}
& \frac{\partial E}{\partial C_{i, j}}=\sum_{L=1}^{o X}\left(\hat{y}_{L}-y_{L}\right) \cdot w_{i+1} \cdot e^{-\frac{1}{2}\left(\frac{\left(x_{L, j}-C_{i, j}\right)^{2}}{\sigma_{i, j}^{2}}\right)} \cdot \frac{x_{L, j}-C_{i, j}}{\sigma_{i, j}^{2}} ; \\
& \frac{\partial E}{\partial \sigma_{i, j}}=\sum_{L=1}^{o X}\left(\hat{y}_{L}-y_{L}\right) \cdot w_{i+1} \cdot e^{-\frac{1}{2}\left(\frac{\left(x_{L, j}-C_{i, j}\right)^{2}}{\sigma_{i, j}^{2}}\right)} \cdot \frac{x_{L, j}-C_{i, j}}{\sigma_{i, j}^{3}} .
\end{aligned}
$$

Расчет новых $C_{i, j}$ и $\sigma_{i, j}$ :

$$
C_{i, j}=C_{i, j}-h \cdot \frac{\partial E}{\partial C_{i, j}}, \quad \sigma_{i, j}=\sigma_{i, j}-h \cdot \frac{\partial E}{\partial \sigma_{i, j}},
$$

где $h$ - это шаг обучения. На этом этапе шаг обучения подбирается.
Первый и второй этапы алгоритма выполняются последовательно до тех пор, пока $E>\delta$, где $\delta$ - заданная точность.

Многократное повторение двух этапов приводит к полному и быстрому обучению сети, особенно при близких к оптимальным значениям начальных параметров радиальных функций.

Далее рассмотрим нечёткие сети ТСК. Нейро-нечёткие системы или нечёткие нейронные сети - это системы из области искусственного интеллекта, которые комбинируют методы искусственных нейронных сетей и систем на нечёткой логике. Одним из видов нечетких нейронных сетей является ТСК пятислойная нейронная сеть, структурная схема которой представлена на рис. 3 [5].

На первом слое вычисляются значения функций принадлежности входных переменных нейронам промежуточного слоя. В каче-

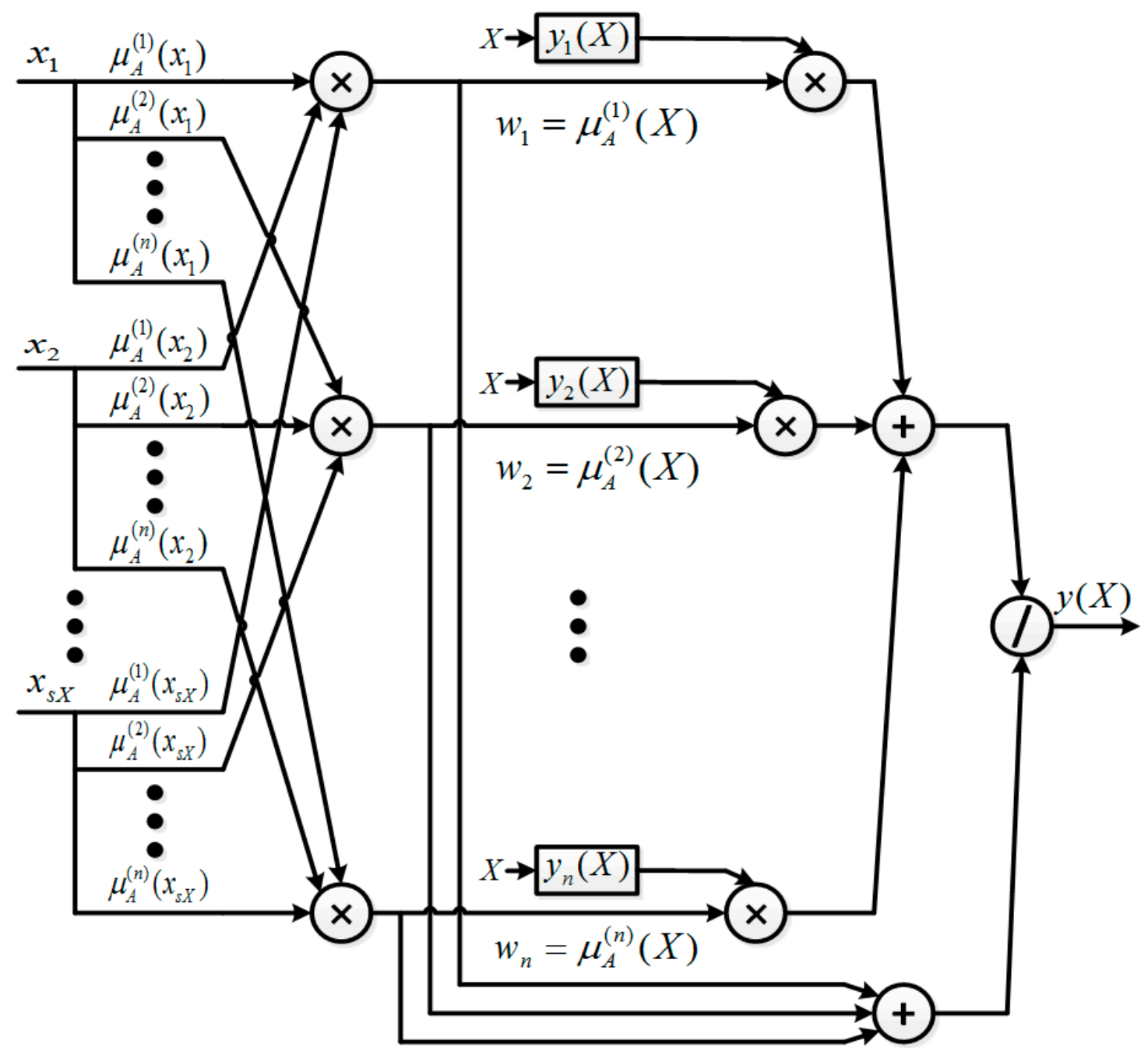

Рис. 3. Обобщённая структура нейронной сети ТСК 


\section{И. А. Седьх, В. А. Истомин}

стве функции фаззификации для каждой входной переменной используется обобщенная функция Гаусса, параметрами которой являются: центр $C$, ширина разброса $\sigma$, степень $b$. На втором слое находятся коэффициенты принадлежности $w_{k}(k=1, \ldots, n)$ входного вектора $X$ нейронам промежуточного слоя. На третьем слое вычисляются линейные параметры $p$ функций ТСК для каждого промежуточного нейрона: $y_{k}(X)=p_{k, 0}+\sum_{t=1}^{s X} p_{k, t} \cdot x_{t}$. Выполняется умножение сигналов $y_{k}(X)$ на $w_{k}$. Четвёртый слой состоит из двух нейронов-сумматоров. Первый - для суммирования $y_{k}(X)$, второй - для суммы весов $w_{k}$. Пятый слой является нормализующим и состоит из одного выходного нейрона. В нем выход первого нейрона-сумматора делится на выход второго, то есть веса подвергаются нормализации [5].

Ниже рассмотрим двухэтапный гибридный алгоритм обучения ТСК. Сначала случайным образом задаются нелинейные параметры сети ТСК: центры, среднеквадратические отклонения, степени функций.

На первом этапе гибридного алгоритма эти значения фиксируются, и по ним подбираются линейные параметры ТСК.

Нормализованные веса нейронов на скрытом слое вычисляются по формуле:

$$
\begin{aligned}
& w_{L, j}=\frac{\mu_{L, j}}{\sum_{z=1}^{n}\left(\mu_{L, z}\right)}, \\
& W=\left[\begin{array}{ccccccccc}
w_{1,1} & w_{1,1} \cdot x_{1,1} & \cdots & w_{1,1} \cdot x_{1, s X} & \cdots & w_{1, n} & w_{1, n} \cdot x_{1,1} & \cdots & w_{1, n} \cdot x_{1, s X} \\
w_{2,1} & w_{2,1} \cdot x_{2,1} & \cdots & w_{2,1} \cdot x_{2, S X} & \cdots & w_{2, n} & w_{2, n} \cdot x_{2,1} & \cdots & w_{2, n} \cdot x_{2, s X} \\
\cdots & \cdots & \cdots & \cdots & \cdots & \cdots & \cdots & \cdots & \cdots \\
w_{o X, 1} & w_{o X, 1} \cdot x_{o X, 1} & \cdots & w_{o X, 1} \cdot x_{o X, S X} & \cdots & w_{o X, n} & w_{o X, n} \cdot x_{o X, 1} & \cdots & w_{o X, n} \cdot x_{o X, s X}
\end{array}\right] \text {, } \\
& P=\left[\begin{array}{c}
p_{1,0} \\
\cdots \\
p_{1, S X} \\
\cdots \\
p_{n, 0} \\
\cdots \\
p_{n, S X}
\end{array}\right], Y=\left[\begin{array}{c}
y_{1} \\
y_{2} \\
\cdots \\
y_{o X}
\end{array}\right] .
\end{aligned}
$$

Рис. 4. Матрицы системы для нахождения линейных параметров ТСК 
где $\frac{\partial E}{\partial C_{j, l}}=\left(\hat{y}_{L}-y_{L}\right) \cdot \sum_{k=1}^{n}\left(p_{k, 0}+\sum_{t=1}^{s X} p_{k, t} \cdot x_{L, t}\right) \cdot \frac{\partial w}{\partial C_{j, l}} ;$ $\sigma_{j, l}=\sigma_{j, l}+h \sigma \cdot \frac{\partial E}{\partial \sigma_{j . l}}$,

где $\frac{\partial E}{\partial \sigma_{j . l}}=\left(\hat{y}_{L}-y_{L}\right) \cdot \sum_{k=1}^{n}\left(p_{k, 0}+\sum_{t=1}^{s X} p_{k, t} \cdot x_{L, t}\right) \cdot \frac{\partial w}{\partial \sigma_{j, l}}$;

$$
b_{j, l}=b_{j, l}+h b \cdot \frac{\partial E}{\partial b_{i, K}},
$$

где $\frac{\partial E}{\partial b_{j, l}}=\left(\hat{y}_{L}-y_{L}\right) \cdot \sum_{k=1}^{n}\left(p_{k, 0}+\sum_{t=1}^{\partial b_{\dot{k} \mathcal{K}}} p_{k, t} \cdot x_{L, t}\right) \cdot \frac{\partial w}{\partial b_{j, l}}$.

На этом этапе шаги обучения $h c, h \sigma, h b$ подбираются.

Первый и второй этапы алгоритма выполняются последовательно до тех пор, пока $E>\delta$.

\section{МОДЕЛИРОВАНИЕ ПРОЦЕССА ОХЛАЖДЕНИЯ ПОЛОСЫ}

Рассмотрим моделирование процесса охлаждения полосы. Для наглядного отображения переменных, участвующих в построении математической модели, приведена табл. 1 с заранее выбранными входными и выходными переменными и пояснениями к ним.

Таблица 1

Входные и выходные переменные

\begin{tabular}{|c|c|}
\hline \multicolumn{2}{|c|}{ Входные переменные } \\
\hline$x_{1}$ & Толщина полосы \\
\hline$x_{2}$ & Ширина полосы \\
\hline$x_{3}-x_{17}$ & Химический состав полосы \\
\hline$x_{18}$ & Температура конца прокатки \\
\hline$x_{19}$ & Количество активных секций \\
\hline$x_{20}$ & Средняя скорость полосы \\
\hline$x_{21}$ & Среднее давление воды \\
\hline$x_{22}$ & Средняя температура воды \\
\hline \multicolumn{2}{|c|}{ Выходные переменные } \\
\hline$y$ & Температуры смотки \\
\hline
\end{tabular}

Кроме того, произведен отбор данных по их принадлежности к участку душирования, выполненный по переменной, отвечающей за положение головы полосы относительно последней клети стана.

Скорость полосы, давление и температуры воды рассчитываются как средние показатели измерений для определенного сечения полосы металла. Таким образом, исходные данные включают 22 входные и 1 выходную переменные [10-14].

Прежде чем перейти к обучению сетей, нужно составить обучающую и проверочную выборки, а затем выполнить нормализацию всех данных.

Формирование выборок для данных по душированию горячекатаной стали произведено с помощью SQL-запросов. Выбраны переменные и отобраны по параметру, который отвечает за показания положения головы листа относительно последней клети. Далее удалены все показания, не относящиеся непосредственно к участку душирования, в результате чего сформирована таблица данных из 2843 строк. На основе полученной таблицы проводится обучение и тестирование нейронных сетей. Для равномерного распределения данных среди обучающей и проверочной выборок каждая десятая строка относится к проверочной выборке, оставшиеся используются для обучений нейронных сетей.

Объём обучающей и проверочной выборок составляет 2559 и 284 строк соответственно. Перед обучением данные нормализуются по формуле:

$$
N_{\text {норм }}=\frac{N-N_{\min }}{N_{\max }-N_{\min }},
$$

где $N$ - значение переменной до нормирования; $N_{\min }$ - минимальное значение по переменной до нормирования; $N_{\max }$ - максимальное значение по переменной до нормирования.

От формирования выборок можно перейти к определению оптимальной структуры и обучению нейронных сетей.

В ходе проведения вычислительных экспериментов на основе разработанных программ были получены следующие оптимальные структуры РБФ и ТСК, приведенные ниже.

Архитектура радиально-базисной нейронной сети: 
- количество нейронов на скрытом слое равно 25;

- количество итераций равно 4;

- начальный шаг обучения центров и радиусов равен 0,5 .

На рис. 5 приведен фрагмент файла, хранящего данные РБФ сети на последней итерации. Ячейки соответствуют следующим переменным: «А2» - номер последней выполненной итерации; «В2» - ошибка обучения нейронной сети; «С2»- количество нейронов на скрытом слое; «D2» - шаг обучения сети на последней итерации; «D4»-коэффициент сравнения ошибок обучения; «D5» - коэффициент замедления шага обучения; «D6»-коэффициент ускорения шага обучения; «Е2»начальный шаг обучения; столбец F, начиная c «F2»- выходные значения обучаемой сети; столбец $\mathrm{G}$, начиная c «G2» - обучающее множество значений выхода.

\begin{tabular}{|c|c|c|c|c|c|c|c|}
\hline 4 & A & $B$ & C & D & $E$ & $\mathrm{~F}$ & G \\
\hline \multicolumn{8}{|l|}{1} \\
\hline 2 & 4 & 19,201 & 25 & 0,0057 & 0,5 & 0,474177 & 0,6271 \\
\hline 3 & & & & & & 0,462444 & 0,5847 \\
\hline 4 & & & & 1 & & 0,478710 & 0,5847 \\
\hline 5 & & & & 0,7 & & 0,475384 & 0,6186 \\
\hline 6 & & & & 1,07 & & 0,527936 & 0,6483 \\
\hline 7 & & & & & & 0,567312 & 0,5508 \\
\hline 8 & & & & & & 0,572055 & 0,5636 \\
\hline$a$ & & & & & & ก 571113 & ก 5678 \\
\hline & , & Sheet1 & Sheet2 & Sheet3 & $\oplus$ & & \begin{tabular}{|l|l|}
1 & \\
\end{tabular} \\
\hline
\end{tabular}

Рис. 5. Фрагмент файла с данными обучения РБФ

Архитектура Такаги - Сугено - Канга:

- количество нейронов на скрытом слое равно 3;

- количество итераций равно 7;

- начальный шаг обучения центров $C_{j, l}$ ТСК функций равен 0,05 ;

- начальный шаг обучения отклонений $\sigma_{j, l}$ ТСК функций равен 0,5 ;

- начальный шаг обучения степени функций $b_{j, l}$ ТСК функций равен 0,5.

Структура файла, хранящего данные ТСК сети на последней итерации, схожа с аналогичным файлом для РБФ. Отличием является отсутствие сохранения шага обучения сети на последней итерации в «D2», так как он не зависит от предыдущих шагов. На каждой итерации подстройка происходит индивидуально для 3-х параметров.

\section{РЕЗУЛЬТАТЫ И ИХ ОБСУЖДЕНИЕ}

После обучения ТСК и РБФ по описанным выше алгоритмам, можно провести их сравнение и выделить основные отличия [19-22].

Ниже приведена таблица сравнения обученных РБФ и ТСК нейронных сетей (табл. 2) по денормализованным данным для обучающей и проверочной выборок.

Таблица 2

Сравнение обученных РБФ

и ТСК нейронных сетей

\begin{tabular}{|c|c|c|c|c|c|c|}
\hline & $n$ & $I$ & $S_{o 6}$ & $S_{n p}$ & $A_{o 6}, \%$ & $A_{n p}, \%$ \\
\hline РБФ & 25 & 4 & 28,91 & 28,65 & 2,67 & 2,57 \\
\hline ТСК & 3 & 7 & 19,12 & 18,79 & 2,02 & 1,75 \\
\hline
\end{tabular}

В табл. 2 приведены сравнения сетей по следующим параметрам: $n$ - количество нейронов на промежуточном слое, $I$ - количество итераций, $S_{o \sigma}, S_{n p}$ - среднеквадратические ошибки обучающей и проверочной выборок соответственно, $A_{o 6}, A_{n p}-$ средние приведенные ошибки [14-15].

Среднеквадратическая ошибка находится по формуле:

$$
S=\sqrt{\frac{1}{o X} \sum_{L=1}^{o X}\left(y_{L}-\hat{y}_{L}\right)^{2}},
$$

где $o X$ - объём выборки; $y_{L}$ - выходное значение; $\hat{y}_{L}-$ выходное значение нейронной сети; $L$ - номер строки данных.

Средняя приведенная ошибка равна:

$$
A=\frac{100 \%}{o X} \sum_{i=1}^{o X} \frac{\left|y_{L}-\hat{y}_{L}\right|}{y_{\max }},
$$

где $y_{\max }=\max _{L} y_{L}$.

На рис. 6 показаны графики сравнения наблюдаемых и предсказанных значений РБФ и ТСК сетей для фрагментов обучающей и проверочной выборок соответственно.

По результатам видно, что ТСК на предоставленном наборе данных обучилась лучше.

При сравнении РБФ и ТСК нейронных сетей можно выделить следующие особенности и отличия:

- РБФ сеть по своей структуре более подходит при обучении простых систем за счет меньшего количества параметров обучения. 

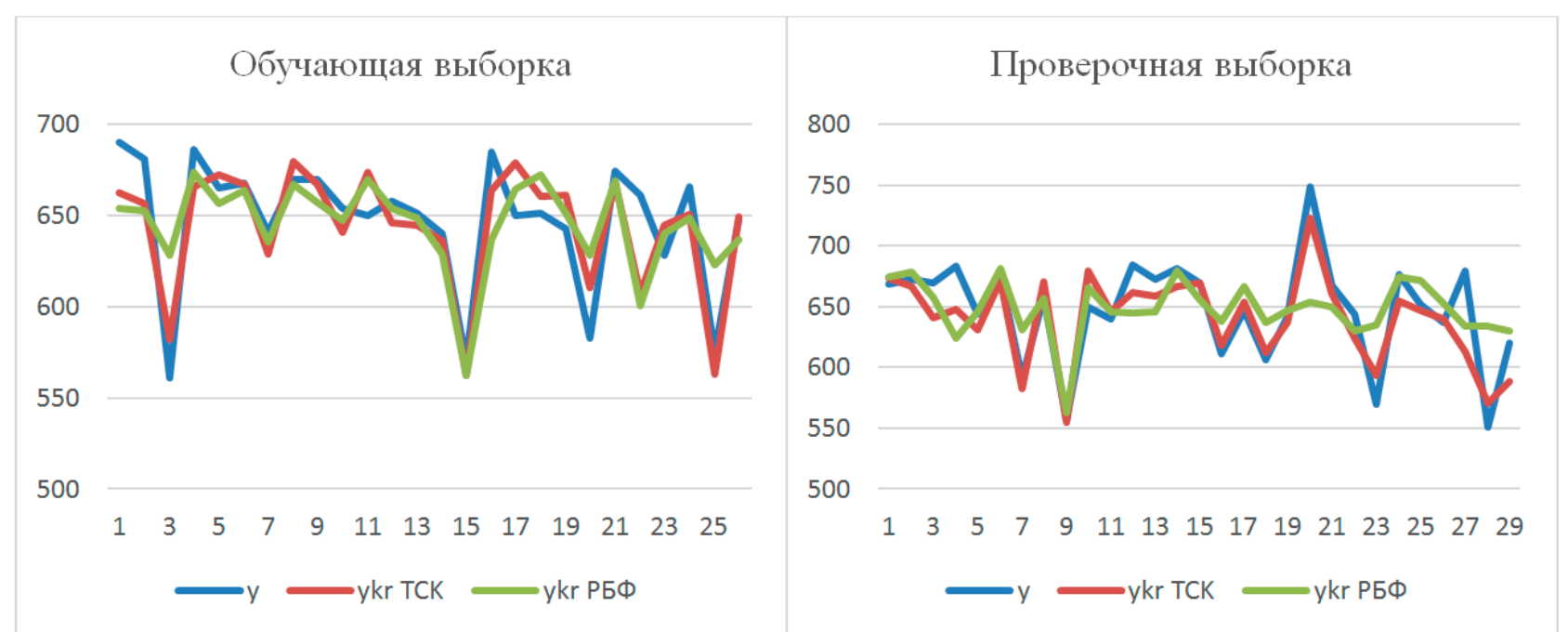

Рис. 6. Сравнение сетей по обучающей и проверочной выборкам

Она позволяет получить приблизительно одинаковые с ТСК результаты обучения при меньшем количестве итераций, но при этом требуется большее количество нейронов на скрытом слое.

- ТСК сеть за счет своей сложной архитектуры, трёх параметров, влияющих на подстройку обучения сети, является более гибкой при обучении различных сложных систем, но для достижения результата приходится опытным путем подбирать все начальные параметры обучаемой сети.

Таким образом, можно сделать вывод, что РБФ и ТСК сети являются по-своему уникальными, выбор между ними зависит от сложности обучаемой системы.

\section{ЗАКЛЮЧЕНИЕ}

В статье рассмотрено нейромоделирование процесса охлаждения полосы на стане горячей прокатки металлургического производства.

На основе реализованных в пакете Mathcad программ построения РБФ и ТСК сетей по производственным данным металлургического производства на участке охлаждения горячекатаной стали разработаны модели процесса охлаждения полосы на стане горячей прокатки металлургического производства. Проведено сравнение моделей с реальными данными, а также сравнение результатов моделирования, полученных на основе
РБФ и ТСК. Данные нейросетевые модели являются имитационными. Они позволяют с достаточной точностью проводить анализ и настройку действующих систем управления процессом душирования, а также прогноз температуры смотки полосы, избегая производственного тестирования. Разработанные модели могут уменьшить затраты при производстве стали на стане горячей прокатки.

Работа выполнена при поддержке РФФИ (проект № 19-48-480007 p_a).

\section{СПИСОК ЛИТЕРАТУРЫ}

1. Линчевскици, Б. В. Металлургия черных металлов / Б. В. Линчевский, А. Л. Соболевский, А. А. Кальменев. - Москва : Металлургия, 1986. - 360 с.

2. Цюрко, В. И. Адаптивный алгоритм управления установкой ускоренного охлаждения горячекатаной полосы / В. И. Цюрко, 3. К. Кабаков // Производство проката. 2011. - №10. - С. 42-45.

3. Гончаров, А. Л. «Основные этапы идентификации системы ускоренного охлаждения проката на основе нейросетевого подхода» / А. Л. Гончаров // Новые информационные технологии в автоматизированных системах. - Москва : Изд-во Миэм НИУ ВШЭ, 2006. - T. 9. - C. 69-55.

4. Сараев, П. В. Перспективы интервального нейросетевого моделирования и про- 
гнозирования / П. В. Сараев, Ю. Е. Полозова // Вестник Липецкого государственного технического университета. - 2016. - №1 (27). C. 6-13.

5. Осовский, С. Нейронные сети для обработки информации / С. Осовский. - Москва : Финансы и статистика, 2002. - 344 с.

6. Погодаев, А. К. Идентификация нейро-нечётких моделей для данных больших объемов / А. К. Погодаев, П. В. Сараев // Вестник Воронежского государственного технического университета. - 2015 - Т. 11, № 4. C. 8-11.

7. Domhan, T. Speeding up automatic hyperparameter optimization of deep neural networks by extrapolation of learning curves / T. Domhan, J. T. Springenberg, F. Hutter // Int. Joint Conf. Articial Intelligence, Buenos Aires, Argentina. 2015. - P. 3460-3468.

8. Блюмин, С. Л. Идентификация параметров входных нечётких процессов разностных нейронечётких переключаемых моделей / С. Л. Блюмин, А. М. Шмырин, Н. Ю. Жбанова // Системы управления и информационные технологии. - 2014. - № 1(55). - С. 8-12.

9. Iofe, S. Batch Normalization: Accelerating Deep Network Training by Reducing Internal Covariate Shif / S. Iofe and C. Szegedy. // Int. Conf. Mach. Learn. Vol. 37. - 2015. - P. 448-456.

10. Истомин, В. А. Обучение нейронных сетей Такаги - Сугно - Канга в программе Mathcadна основе гибридного алгоритма / В. А. Истомин, И. А. Седых // Сборник научных статей IV научно-практической международной конференции (школы-семинара) молодых ученых (23-25 апреля 2018 г.). В двух частях. - Тольятти : Издатель Качалин Александр Васильевич, 2018. Ч. 2. - С. 407-411.

11. Кластеризация обучающих выборок большого объёма для обучения нейронных сетей на репрезентативных подвыборках / М. Г. Журавлева [и др.] // Вести высших учебных заведений Черноземья. - 2015. - № 1. C. 54-61.

12. Sedykh, I. A. Neural Networks Neighborhood Models / I. A. Sedykh, A. M.Shmyrin // Global Journal of Pure and Applied Mathematics. ISSN 0973-1768 Volume 12, Number 6 (2016). P. 5039-5046.
13. Krizhevsky, A. Imagenet classification with deep convolutional neural networks. In Advances in neural information processing systems / A. Krizhevsky, I. Sutskever, G. E. Hinton. 2012. - P. 1097-1105.

14. Барский, А. Б. Логические нейронные сети: учебное пособие / А. Б. Барский. - Москва : Бином, 2013. - 352 с.

15. Яхъяева, Г. Э. Нечеткие множества и нейронные сети: учебное пособие / Г. Э. Яхъяева. - Москва : Национальный Открытый Университет «Интуит», 2016. - 187 с.

16. Седых, И. А. Гибкое управление светофорной системой перекрестка на основе нейронных сетей / И. А. Седых, Д. С. Демахин // Автоматизация процессов управления. 2017. - № 1(47). - С. 94-100.

17. Седых, И. А. Исследование, анализ и обработка данных с помощью искусственных нейронных сетей в программе STATISTICA / И. А. Седых, В. А. Истомин // Вестник Липецкого государственного технического университета. - 2017. - №1 (31). - C. 33-37.

18. Jozefowicz, R. An Empirical Exploration of Recurrent Network Architectures / R. Jozefowicz, W. Zaremba, and I. Sutskever // Int. Conf. Mach. Learn. - 2015. - Vol. 37. PMLR. P. 2342-2350.

19. Ursino, M. Multisensory Bayesian inference depends on synapse maturation during training: Theoretical analysis and neural modeling implementation / M. Ursino, C. Cuppini, and E. Magosso // Neural Computation. - 2017. vol. 29, no. 3, pp. 735-782.

20. Сараев, П. В. Применение результатов интервального нейросетевого прогнозирования для калибровки средств измерений в системах управления / П. В. Сараев, Ю. Е. Полозова, Ю. Л. Полозов // Проблемы управления. - 2017. - № 2. - С. 50-55.

21. Сараев, П.В. Ремоделирование и трансформация моделей в нейро-нечеткие / П. В. Сараев, А. В. Галкин // Наукоемкие технологии и инновации международная научно-практическая конференция (XXII научные чтения). - 2016. - С. 62-66.

22. Pogodaev, A. Neurostructural modelling and prediction of hot-rolled production defects by casting parameters / A. Pogodaev, P. Saraev // Journal of Chemical Technology and Metallurgy. - 2015. - T. 50, № 6. - P. 595-599. 
Седых Ирина Александровна - канд. физ.мат. наук, доцент, доцент кафедры высшей математики ФГБОУ ВО «Липецкий государственный технический университет», Россия, Липецк. E-mail: sedykh-irina@yandex.ru
Истомин Владимир Александрович - магистрант ФГБОУ ВО «Липецкий государственный технический университет», Россия, Липецк.

E-mail: istomin96@mail.ru

\title{
NEURO NETWORK MODELING OF THE STRIP COOLING PROCESS AT THE HOT ROLLING STATION
}

\author{
I. A. Sedykh, V. A. Istomin \\ Lipetsk State Technical University
}

\begin{abstract}
Annotation. In the work, the process of strip cooling in the hot rolling mill of metallurgical production is considered and modeled on the basis of neural networks. This process is schematically depicted in the figure, highlighting the main functional areas. Clarified basic terms related to the above process of metallurgical production. The block diagrams of the radial-basic neural network and the Takagi - Sugeno - Kang neural network are shown, and hybrid learning algorithms are described. In the Mathcad programming unit, programs have been developed that implement the mathematical models of the considered neural networks. Input and output data for programs are respectively read and saved in Microsoft Excel files. The input and output variables of the modeled process are selected, the linear normalization of the initial data is performed. Formed training and test samples using queries in Microsoft Access. For these models, a number of computational experiments were performed, during which the optimal structure and parameters of the radial-based neural network and the Takagi - Sugeno - Kang neural network were obtained. A comparison of the trained neural networks is carried out, graphs of deviations of the training and test samples, as well as the results of their training are given. The root-mean-square and relative errors of the networks are found from the denormalized output data. Based on the analysis performed, the main features and differences of the above neural networks are highlighted. Conclusions on the work done.

Keywords: modeling, radial-basic neural networks, Takagi - Sugeno - Kang neural networks, training of neural networks, strip cooling process, neural network architecture.
\end{abstract}

Sedykh I. A. - candidate of Physics and Mathematics, Associate Professor, Associate Professor of the Mathematics Department at the Lipetsk State Technical University, Russia, Lipetsk.

E-mail: sedykh-irina@yandex.ru
Istomin V. A. - smaster student of Lipetsk State Technical University, Russia, Lipetsk. E-mail: sedykh-irina@yandex.ru 